Georgian Mathematical Journal

1(1994), No. 3, 235-242

\title{
ON CONVERGENCE SUBSYSTEMS OF ORTHONORMAL SYSTEMS
}

\author{
G. BARELADZE
}

\begin{abstract}
It is proved that for any sequence $\left\{R_{k}\right\}_{k=1}^{\infty}$ of real numbers satisfying $R_{k} \geq k(k \geq 1)$ and $R_{k}=o\left(k \log _{2} k\right), k \rightarrow \infty$, there exists a orthonormal system $\left\{\varphi_{n}(x)\right\}_{n=1}^{\infty}, x \in(0 ; 1)$, such that none of its subsystems $\left\{\varphi_{n_{k}}(x)\right\}_{k=1}^{\infty}$ with $n_{k} \leq R_{k}(k \geq 1)$ is a convergence subsystem.
\end{abstract}

Let $\left\{\varphi_{n}(x)\right\}$ be an orthonormal system (ONS) on $(0 ; 1)$. It is called a convergence system if the series $\sum c_{n} \varphi_{n}(x)$ is convergent almost everywhere whenever the sequence $\left\{c_{n}\right\}$ of real numbers satisfies $\sum c_{n}^{2}<\infty$.

It is well-known [1] that not every ONS $\left\{\varphi_{n}(x)\right\}$ is a convergence system. However [2], [3], each of them contains some convergence subsystem $\left\{\varphi_{n_{k}}(x)\right\}$. A question was formulated later [4] whether there exists a common estimate of growth rate of numbers $n_{k}$ in the class of all ONS. B.S.Kashin [5] answered this question in the affirmative: one can determine a sequence of positive numbers $\left\{R_{k}\right\}$ such that from any ONS it is possible to choose a convergence subsystem $\left\{\varphi_{n_{k}}\right\}$ with $n_{k} \leq R_{k}, 1 \leq k<\infty$. In the same paper [5] the problem of finding $\left\{R_{k}\right\}$ with a minimal admissible growth order is formulated and the hypothesis $R_{k}=k^{1+\varepsilon}(\varepsilon>0)$ is conjectured. G.A.Karagulyan [6] proved that one can take $R_{k}=\lambda^{k}, \lambda>1$. However, this upper estimate is rougher than the one expected in [5].

In this paper we shall give the proof of the theorem providing the lower estimate for $\left\{R_{k}\right\}$.

Theorem. For any sequence $\left\{R_{k}\right\}_{k=1}^{\infty}$ of real numbers satisfying

$$
R_{k} \geq k(k \geq 1) \text { and } R_{k}=o\left(k \log _{2} k\right), \quad k \rightarrow \infty,
$$

there exists an $\operatorname{ONS}\left\{\varphi_{n}(x)\right\}_{n=1}^{\infty}, x \in(0 ; 1)$, such that none of its subsystems $\left\{\varphi_{n_{k}}(x)\right\}_{k=1}^{\infty}$ with $n_{k} \leq R_{k}(k \geq 1)$ is a convergence subsystem.

Several lemmas are needed to prove this theorem.

1991 Mathematics Subject Classification. 42C20. 
Lemma 1 (H.Rademacher [7]). For any ONS $\left\{\psi_{n}(x)\right\}_{n=1}^{N}, x \in(0 ; 1)$, and any collection of real numbers $\left\{c_{n}\right\}_{n=1}^{N}$

$$
\int_{0}^{1}\left(\max _{1 \leq j \leq N}\left|\sum_{n=1}^{j} c_{n} \psi_{n}(x)\right|\right)^{2} d x \leq c \log _{2}^{2}(N+1) \sum_{n=1}^{N} c_{n}^{2}, \quad 1 \leq N<\infty .^{1}
$$

Lemma 2. For any $N \geq 1$ there exists an $O N S$

$$
\psi(N):=\left\{\psi_{n}^{N}(x)\right\}_{n=1}^{N}, \quad x \in(0 ; 1),
$$

satisfying, for any collection of natural numbers $1 \leq n_{1}<n_{2}<\cdots<n_{m} \leq$ $N(1 \leq m \leq N)$, the inequality

$$
\int_{0}^{1} \max _{1 \leq j \leq m}\left|\sum_{k=1}^{j} \psi_{n_{k}}^{N}(x)\right| d x \geq c \frac{\log _{2} N}{\sqrt{N}} m .
$$

Proof. We shall assure that the requirements of this lemma are satisfied by the ONS usually used in the proof of the Menshov-Rademacher theorem. The functions $\psi_{n}^{N}(x), 1 \leq n \leq N$, belonging to this ONS (see [8], p.295) have, in particular, the following properties:

(i)

$$
\psi_{n}^{N}(x):= \begin{cases}\frac{c \sqrt{N}}{s-n}, & x \in\left(\frac{s-1}{2 N} ; \frac{2 s-1}{4 N}\right), \\ \frac{c \sqrt{N}}{n-s}, & x \in\left(\frac{2 s-1}{4 N} ; \frac{s}{2 N}\right), \quad 1 \leq s \leq N, \quad s \neq n \\ 0, & x \in\left(\frac{n-1}{2 N} ; \frac{n}{2 N}\right) ;\end{cases}
$$

(ii) $\psi_{n}^{N}(x)$ is constant on each of the intervals

$$
\left(\frac{s-1}{4 N} ; \frac{s}{4 N}\right), \quad 2 N+1 \leq s \leq 4 N
$$

(iii)

$$
\int_{0}^{1} \psi_{n}^{N}(x) d x=0
$$

(iv) $\psi_{n}^{N}(x)$ is extended from $(0 ; 1)$ onto $(-\infty ; \infty)$ with period 1 .

Denote $\delta_{s}:=\left(\frac{s-1}{2 N} ; \frac{2 s-1}{4 N}\right), 1 \leq s \leq N$. When $x \in \delta_{s}$, because of (i) we have $\psi_{p}^{N}(x) \geq 0$ for $1 \leq p \leq s$ and $\psi_{p}^{N}(x) \leq 0$ for $s \leq p \leq N(1 \leq s \leq N)$.

\footnotetext{
${ }^{1}$ Here and in what follows $c$ denotes positive absolute constants which, in general, may differ from one equality (inequality) to another.
} 
Therefore for fixed numbers $1 \leq n_{1}<n_{2}<\cdots<n_{m} \leq N(1 \leq m \leq N)$ and for each $x \in \delta_{s}(1 \leq s \leq N)$ we obtain

$$
\begin{gathered}
\sum_{k=1}^{m}\left|\psi_{n_{k}}^{N}(x)\right|=\sum_{k: n_{k} \leq s}+\sum_{k: n_{k}>s}=\left|\sum_{k: n_{k} \leq s} \psi_{n_{k}}^{N}(x)\right|+ \\
+\left|\sum_{k: n_{k}>s} \psi_{n_{k}}^{N}(x)\right| \leq 3 \max _{1 \leq j \leq m}\left|\sum_{k=1}^{j} \psi_{n_{k}}^{N}(x)\right| .
\end{gathered}
$$

Hence

$$
\begin{gathered}
\int_{\delta_{s}} \max _{1 \leq j \leq m}\left|\sum_{k=1}^{j} \psi_{n_{k}}^{N}(x)\right| d x \geq \frac{1}{3} \int_{\delta_{s}} \sum_{k=1}^{m}\left|\psi_{n_{k}}^{N}(x)\right| d x= \\
=\frac{c}{\sqrt{N}} \sum_{\substack{k: 1 \leq k \leq m, n_{k} \neq s}} \frac{1}{\left|n_{k}-s\right|}, \quad 1 \leq s \leq N ; \\
\int_{0}^{1} \max _{1 \leq j \leq m}\left|\sum_{k=1}^{j} \psi_{n_{k}}^{N}(x)\right| d x \geq \sum_{s=1}^{N} \int_{\delta_{s}} \max _{1 \leq j \leq m}\left|\sum_{k=1}^{j} \psi_{n_{k}}^{N}(x)\right| d x \geq \\
\geq \frac{c}{\sqrt{N}} \sum_{s=1}^{N} \sum_{\substack{k: 1 \leq k \leq m \\
n_{k} \neq s}} \frac{1}{\left|n_{k}-s\right|}= \\
=\frac{c}{\sqrt{N}} \sum_{k=1}^{m} \sum_{\substack{k: 1 \leq s \leq N, s \neq n_{k}}} \frac{1}{\left|n_{k}-s\right|} \geq c \frac{\log _{2} N}{\sqrt{N}} m .
\end{gathered}
$$

Remark 1. For any positive integer $Q,\left\{\psi_{n}^{N}(Q x)\right\}_{n=1}^{N}$ is an ONS on $(0 ; 1)$ also satisfying the inequality (2).

Remark 2. Let $N_{0}, N_{1}, Q_{0}, Q_{1}, p$ be positive integers. If $Q_{1}=4 p N_{0} Q_{0}$, then functions belonging to different collections $\left\{\psi_{n}^{N_{0}}\left(Q_{0} x\right)\right\}_{n=1}^{N_{0}}$ and $\left\{\psi_{n}^{N_{1}}\left(Q_{1} x\right)\right\}_{n=1}^{N_{1}}$ are mutually orthogonal and pairwise stochastically independent on $(0 ; 1)$.

Both conclusions follow readily from (i)-(iv).

Lemma 3. Suppose a function $f \in L_{(0 ; 1)}^{1}$ is not equivalent to zero and

$$
A:=\left\{x \in(0 ; 1):|f(x)|>\frac{1}{2}\|f\|_{L_{(0 ; 1)}^{1}}\right\} .
$$

Then

$$
\operatorname{mes} A \geq\|f\|_{L_{(0 ; 1)}^{1}}^{2} / 4\|f\|_{L_{(0 ; 1)}^{2}}^{2}
$$


Proof. Indeed, we obtain (using Hölder's inequality)

$$
\begin{aligned}
\|f\|_{L_{(0 ; 1)}^{1}} & =\int_{A}|f(x)| d x+\int_{(0 ; 1) \backslash A}|f(x)| d x \leq \\
& \leq(\operatorname{mes} A)^{1 / 2} \cdot\|f\|_{L_{(0 ; 1)}^{2}}+\frac{1}{2}\|f\|_{L_{(0 ; 1)}^{1}},
\end{aligned}
$$

which immediately implies (3).

Lemma 4. Let $N, Q, m(1 \leq m \leq N)$ be positive integers. Then for any collection of natural numbers $1 \leq n_{1}<n_{2}<\cdots<n_{m} \leq N$

$$
\operatorname{mes}\left\{x \in(0 ; 1): \max _{1 \leq j \leq m}\left|\sum_{k=1}^{j} \psi_{n_{k}}^{N}(Q x)\right|>\frac{J}{2}\right\} \geq c \frac{m}{N},
$$

where $\psi_{n}^{N}(x) \in \psi(N), 1 \leq n \leq N$ (see Lemma 2), and

$$
J:=\int_{0}^{1} \max _{1 \leq j \leq m}\left|\sum_{k=1}^{j} \psi_{n_{k}}^{N}(Q x)\right| d x .
$$

Proof. By Lemmas 1 and 2 (see also Remark 1) we have

$$
\begin{gathered}
\int_{0}^{1}\left(\max _{1 \leq j \leq m}\left|\sum_{k=1}^{j} \psi_{n_{k}}^{N}(Q x)\right|\right)^{2} d x \leq c m \log _{2}^{2}(m+1), \\
J^{2} \geq c \frac{m^{2}}{N} \log _{2}^{2} N ; \quad 1 \leq m \leq N<\infty .
\end{gathered}
$$

Thus, applying Lemma 3 , we obtain

$\operatorname{mes}\left\{x \in(0 ; 1): \max _{1 \leq j \leq m}\left|\sum_{k=1}^{j} \psi_{n_{k}}^{N}(Q x)\right|>\frac{J}{2}\right\} \geq c \frac{m}{N} \cdot \frac{\log _{2}^{2} N}{\log _{2}^{2}(m+1)} \geq c \frac{m}{N}$.

Proof of the theorem. If the conditions (1) are fulfilled, then $R_{k}=k \log _{2} k / \varepsilon(k)$, $2 \leq k<\infty$, where $\varepsilon(k)$ tends to infinity. Moreover, without loss of generality, it can be assumed that

a) $R_{1}=1$

b) $\varepsilon(k)$ is a nondecreasing sequence of positive integers;

c) the sets $\Delta_{m}:=\{k: \varepsilon(k)=m\}$ have the form $\left(\nu_{m-1} ; \nu_{m}\right] \cap \mathbb{N}$ $(m=1,2, \ldots)$, where $\nu_{0}=0, \log _{2} \log _{2} \log _{2} \nu_{m}=p_{m}(m \geq 1)$ and $\left\{p_{m}\right\}_{m=1}^{\infty}$ is some increasing sequence of positive integers.

In particular, for $k \in \Delta_{m}(m \geq 2)$ we have

$$
\begin{gathered}
\log _{2} k>\log _{2} \nu_{m-1}=2^{2^{p_{m-1}}} \geq 2^{2^{m-1}}>m=\varepsilon(k), \\
\varepsilon\left(k \log _{2} k\right) \leq \varepsilon\left(\nu_{m} \log _{2} \nu_{m}\right)<\varepsilon\left(\nu_{m+1}\right)=m+1 \leq 2 \varepsilon(k) .
\end{gathered}
$$


Denote

$$
T_{1}:=0, \quad T_{k}:=2^{\left[k \log _{2} k \log _{2} \log _{2} k\right]}, \quad 2 \leq k<\infty,
$$

and $E_{m}:=\left\{k: R_{k} \leq T_{m}\right\}, 2 \leq m<\infty$ (here $[x]$ is the integer part of the number $x$ ).

Since the function $\varphi(x)=x \varepsilon(x) / \log _{2} x$ increases on $(e ; \infty)$, taking into account (4) and (5), we have, for $m>\nu_{1}$,

$$
\begin{aligned}
E_{m} & =\{1 ; 2\} \cup\left\{k \geq 3: R_{k} \leq T_{m}\right\}=\{1 ; 2\} \cup\left\{k \geq 3: \frac{k \log _{2} k}{\varepsilon(k)} \leq T_{m}\right\}= \\
& =\{1 ; 2\} \cup\left\{k \geq 3: \varphi\left(\frac{k \log _{2} k}{\varepsilon(k)}\right) \leq \varphi\left(T_{m}\right)\right\} \supset \\
& \supset\{1 ; 2\} \cup\left\{k \geq 3: 2 k \leq \varphi\left(T_{m}\right)\right\}=\left\{k \geq 1: k \leq \frac{1}{2} \varphi\left(T_{m}\right)\right\} .
\end{aligned}
$$

Therefore for a large $m\left(m \geq m_{1}>\nu_{1}\right)$

$$
\begin{gathered}
\left|E_{m}\right|>\frac{1}{2} \varphi\left(T_{m}\right)-1 \geq \frac{1}{3} \frac{T_{m} \varepsilon\left(T_{m}\right)}{\log _{2} T_{m}} \\
\left|E_{m+1}\right| \geq \frac{1}{3} \frac{T_{m+1} \varepsilon\left(T_{m+1}\right)}{\log _{2} T_{m+1}} \geq \frac{1}{3} \frac{T_{m+1}}{T_{m} \log _{2} T_{m+1}} \cdot T_{m} \geq 2 T_{m}
\end{gathered}
$$

Because of

$$
\sum_{k \in \Delta_{m}} \frac{1}{\log _{2} T_{k}}=\sum_{k \in \Delta_{m}} \frac{1}{\left[k \log _{2} k \log _{2} \log _{2} k\right]}>\frac{1}{8}\left(p_{m}-p_{m-1}\right) \geq \frac{1}{8} \quad(m \geq 2)
$$

we can select a subsequence $T_{q_{k}} \equiv \widetilde{T}_{k}\left(1 \leq k<\infty ; \widetilde{T}_{1}=0, \widetilde{T}_{2}=\nu_{1}\right)$ such that

$$
\frac{1}{2 m^{2}}<\sum_{k: q_{k} \in \Delta_{m}} \frac{1}{\log _{2} \widetilde{T}_{k}} \equiv \sum_{k: q_{k} \in \Delta_{m}} \frac{1}{\log _{2} T_{q_{k}}} \leq \frac{1}{m^{2}}, \quad m \geq 2,
$$

and hence

$$
\begin{gathered}
\sum_{k: q_{k} \in \Delta_{m}} \frac{\varepsilon\left(\widetilde{T}_{k}\right)}{\log _{2} \widetilde{T}_{k}}=\sum_{k: q_{k} \in \Delta_{m}} \frac{\varepsilon\left(T_{q_{k}}\right)}{\log _{2} T_{q_{k}}} \geq \\
\geq \sum_{k: q_{k} \in \Delta_{m}} \frac{\varepsilon\left(q_{k}\right)}{\log _{2} T_{q_{k}}}=m \sum_{k: q_{k} \in \Delta_{m}} \frac{1}{\log _{2} T_{q_{k}}}>\frac{1}{2 m}, \quad m \geq 2 .
\end{gathered}
$$

Let

$$
\begin{aligned}
N_{m} & :=\widetilde{T}_{m}-\widetilde{T}_{m-1}, \\
Q_{2}:=1, \quad Q_{m+1} & :=4 N_{m} Q_{m}, \quad 2 \leq m<\infty .
\end{aligned}
$$


Consider the orthonormal collections

$$
\left\{\psi_{n}^{N_{m}}\left(Q_{m} x\right)\right\}_{n=1}^{N_{m}}, \quad 2 \leq m<\infty,
$$

and construct with their aid the desired ONS $\left\{\varphi_{n}(x)\right\}_{n=1}^{\infty}$ as

$$
\varphi_{n}(x):=\psi_{k}^{N_{m}}\left(Q_{m} x\right),
$$

where $n \in\left(\widetilde{T}_{m-1} ; \widetilde{T}_{m}\right], k=n-\widetilde{T}_{m-1}, 2 \leq m<\infty, x \in(0 ; 1)$ (the orthonormality follows from (10) and Remark 2).

Let $\left\{n_{k}\right\}_{k=1}^{\infty}$ be a sequence of positive integers with $k \leq n_{k} \leq R_{k}, 1 \leq$ $k<\infty$. We set

$$
\begin{aligned}
& G_{m}:=\left\{k: \widetilde{T}_{m-1}<n_{k} \leq \widetilde{T}_{m}\right\}, \\
& M_{m}:=\left|G_{m}\right|, \\
& a_{n}:=\left(\left(1+M_{m}\right) \log _{2} \widetilde{T}_{m}\right)^{-1 / 2} \text { for } n \in\left(\widetilde{T}_{m-1} ; \widetilde{T}_{m}\right] ; m \geq 2 .
\end{aligned}
$$

On account of (8)

$$
\begin{aligned}
\sum_{k=1}^{\infty} a_{n_{k}}^{2} & =\sum_{m=2}^{\infty} \sum_{k: \widetilde{T}_{m-1}<n_{k} \leq \widetilde{T}_{m}} a_{n_{k}}^{2}=\sum_{m=2}^{\infty} \frac{M_{m}}{\left(1+M_{m}\right) \log _{2} \widetilde{T}_{m}}< \\
& <\sum_{k=2}^{\infty} \frac{1}{\log _{2} T_{q_{k}}}=\sum_{m=2}^{\infty} \sum_{k: q_{k} \in \Delta_{m}} \frac{1}{\log _{2} T_{q_{k}}}<\infty .
\end{aligned}
$$

It is thus sufficient to show that the series

$$
\sum_{k=1}^{\infty} a_{n_{k}} \varphi_{n_{k}}(x)
$$

diverges on some set of positive measure.

Note that

$$
\begin{gathered}
G_{m}=\left\{k: n_{k} \leq \widetilde{T}_{m}\right\} \backslash\left\{k: n_{k} \leq \widetilde{T}_{m-1}\right\} \supset \\
\supset\left\{k: R_{k} \leq \widetilde{T}_{m}\right\} \backslash\left\{k: k \leq \widetilde{T}_{m-1}\right\}, \quad m \geq 2 .
\end{gathered}
$$

Consequently in view of (6) and (7)

$$
\begin{aligned}
M_{m} & \geq\left|\left\{k: R_{k} \leq \widetilde{T}_{m}\right\}\right|-\left|\left\{k: k \leq \widetilde{T}_{m-1}\right\}\right|=\left|\left\{k: R_{k} \leq T_{q_{m}}\right\}\right|-T_{q_{m-1}}> \\
& >\left|E_{q_{m}}\right|-\frac{1}{2}\left|E_{q_{m-1}+1}\right| \geq \frac{1}{2}\left|E_{q_{m}}\right| \geq \frac{1}{6} \frac{\widetilde{T}_{m} \varepsilon\left(\widetilde{T}_{m}\right)}{\log _{2} \widetilde{T}_{m}}, \quad m \geq m_{1} .
\end{aligned}
$$


Hence by (11) and Lemma 2

$$
\begin{aligned}
\widetilde{J}_{m}: & =\int_{0}^{1} \max _{\widetilde{T}_{m-1}<j \leq \widetilde{T}_{m}}\left|\sum_{k: \widetilde{T}_{m-1}<n_{k} \leq j} a_{n_{k}} \varphi_{n_{k}}(x)\right| d x \geq \\
& \geq c \cdot \frac{1}{\sqrt{\left(1+M_{m}\right) \log _{2} \widetilde{T}_{m}}} \cdot \frac{\log _{2} N_{m}}{\sqrt{N_{m}}} \cdot M_{m} \geq \\
& \geq c \sqrt{\frac{M_{m} \log _{2} \widetilde{T}_{m}}{\widetilde{T}_{m}}} \geq c \sqrt{\varepsilon\left(\widetilde{T}_{m}\right)}, \quad m \geq m_{1},
\end{aligned}
$$

and therefore

$$
\lim _{m \rightarrow \infty} \widetilde{J}_{m}=\infty
$$

If $A_{m}$ denotes the set

$$
\left\{x \in(0 ; 1): \max _{\widetilde{T}_{m-1}<j \leq \widetilde{T}_{m}}\left|\sum_{k: \widetilde{T}_{m-1}<n_{k} \leq j} a_{n_{k}} \varphi_{n_{k}}(x)\right|>\frac{1}{2} \widetilde{J}_{m}\right\}, \quad m \geq 2,
$$

then by (11), Lemma 4, (13) and (9)

$$
\begin{gathered}
\operatorname{mes} A_{m} \geq \frac{M_{m}}{N_{m}}>\frac{M_{m}}{\widetilde{T}_{m}}>\frac{1}{6} \frac{\varepsilon\left(\widetilde{T}_{m}\right)}{\log _{2} \widetilde{T}_{m}}, \quad m \geq m_{1} ; \\
\sum_{m=2}^{\infty} \operatorname{mes} A_{m} \geq \frac{1}{6} \sum_{k=m_{1}}^{\infty} \frac{\varepsilon\left(\widetilde{T}_{k}\right)}{\log _{2} \widetilde{T}_{k}}=\frac{1}{6} \sum_{k: q_{k} \geq q_{m_{1}}} \frac{\varepsilon\left(T_{q_{k}}\right)}{\log _{2} T_{q_{k}}}> \\
>\frac{1}{6} \sum_{k: q_{k}>\nu_{m_{1}}} \frac{\varepsilon\left(T_{q_{k}}\right)}{\log _{2} T_{q_{k}}}=\frac{1}{6} \sum_{m=1+m_{1}} \sum_{k: q_{k} \in \Delta_{m}} \frac{\varepsilon\left(T_{q_{k}}\right)}{\log _{2} T_{q_{k}}}=\infty .
\end{gathered}
$$

It is easy to verify (see $(10),(11)$, Remark 2 ) that $\left\{A_{m}\right\}_{m=2}^{\infty}$ is a sequence of stochastically independent sets. Therefore by (15) and the Borel-Cantelli lemma

$$
\operatorname{mes}\left(\lim _{m \rightarrow \infty} \sup A_{m}\right)=1 .
$$

Hence we conclude because of (14) and the definition of sets $A_{m}$ that the series (12) diverges almost everywhere on $(0 ; 1)$.

\section{REFERENCES}

1. D.E. Menshov, Sur les séries des fonctions orthogonales I. Fund. Math. 4(1923), 82-105. 
2. J. Marcinkiewicz, Sur la convergence des séries orthogonales. Studia Math. 6(1936), 39-45.

3. D.E. Menshov, Sur la convergence et la sommation des séries des fonctions orthogonales. Bull. Soc. Math. de France 64(1936), 147-170.

4. G. Bennet, Lectures on matrix transformation of $l_{p}$ spaces. Notes in Banach spaces, pp. 39-80, Univ. Texas Press, Austin, Tex., 1980.

5. B.S. Kashin, The choice of convergence subsystem from the given orthonormal system. (Russian) Uspehi Mat. Nauk 40(1985), No.2(242), 181-182. English translation: Russ. Math. Surv. 40(1985), 215-216.

6. G.A. Karagulyan, The choice of convergence subsystem with logarithmic density from an arbitrary orthonormal system. (Russian) Mat. Sb. (N.S.) 136(178)(1988), No.1, 41-45.

7. H. Rademacher, Einige Sätze über Reihen von allgemeinen Orthogonalfunktionen. Math. Ann. 87(1922), 112-138.

8. B.S. Kashin, A.A. Saakyan, Orthogonal series. (Russian) "Nauka", Moscow, 1984. English translation: Translations of Mathematical Monographs, 75, American Math. Society, Providence, RI, 1989.

(Received 25.02.1993)

Author's address:

Faculty of Mechanics and Mathematics

I. Javakhishvili Tbilisi State University

2, University St., 380043 Tbilisi

Republic of Georgia 